\title{
Conocimientos, experiencias Y actitudes sobre derechos sexuales $Y$ derechos reproductivos en adolescentes $\Psi$ jóvenes
}

\author{
Sara María Torres-Ospina* \\ Ángela María Delgadillo-Morales** \\ Linda Teresa Orcasita-Pineda***
}

Recibido en 28 de junio de 2019, aceptado en 31 de octubre de 2020

Citar este artículo así:

Torres-Ospina SM, Delgadillo-Morales AM, Orcasita-Pineda LT. Conocimientos, experiencias y actitudes sobre derechos sexuales y derechos reproductivos en adolescentes y jóvenes. Hacia. Promoc. Salud. $2021 ; 26$ (1): $52-$ 68 DOI: $10.17151 /$ hpsal.2021.26.1.6

\section{Resumen}

El Modelo de los Servicios de Salud Amigables para Adolescentes y Jóvenes (SSAAJ) corresponde a un proceso creado para garantizar que las instituciones de salud generen espacios y formas de atención integral y diferencial para la población entre 10 y 29 años, permitiendo que se garanticen los Derechos Sexuales y Derechos Reproductivos (DSDR) acorde a las necesidades y expectativas en salud que les afectan. Objetivo: indagar los conocimientos, actitudes y experiencias en DSDR en un grupo de adolescentes y jóvenes vinculados a los SSAAJ. Materiales y métodos: mediante un estudio cualitativo con diseño fenomenológico se realizaron 13 entrevistas semiestructuradas a los adolescentes y jóvenes entre los 13 y 21 años. Resultados: en conocimientos frente a los DSDR los adolescentes hombres tienen algunas nociones sobre derechos humanos, pero desconocen los diferentes DSDR que existen, en las mujeres predomina mayor conocimiento sobre los DSDR. Las actitudes de los adolescentes frente a los DSDR son favorables una vez identifican lo que representan en el abordaje de la sexualidad, sin embargo, consideran que sus experiencias sobre el ejercicio de los DSDR se ven limitadas para su exigibilidad y garantía por el desconocimiento que tienen de ellos. Conclusiones: se encontró que los conocimientos, actitudes y experiencias de los adolescentes y jóvenes sobre los DSDR están influenciados por factores familiares y culturales que impactan sus trayectorias de vida.

\section{Palobras clave}

Sexualidad, derechos sexuales y reproductivos, servicios de salud, adolescente, servicios de salud del adolescente (Fuente: DeCS, BIREME).

\footnotetext{
*Psicóloga. Pontificia Universidad Javeriana Seccional Cali. Santiago de Cali, Colombia. Correo electrónico: saramaria@javerianacali.edu.co (D) orcid.org/0000-0003-2948-6692

"Psicóloga. Pontificia Universidad Javeriana Seccional Cali. Santiago de Cali, Colombia. Correo electrónico: ange2512@javerianacali.edu.co (D) orcid.org/0000-0001-6585-9164

* Magister en Familia. Docente del Departamento de Ciencias Sociales Facultad de Humanidades y Ciencias Grupo de Investigación Bienestar, Trabajo, Cultura y Sociedad. Pontificia Universidad Javeriana Seccional Cali. Santiago de Cali, Colombia. Autor para correspondencia. Correo electrónico: 1torcasita@javerianacali.edu.co@orcid.org/0000-0002-7599-9280 Google
} 


\title{
Knowledge, experiences and attitudes about sexual rights and reproductive rights in adolescents and young people
}

\begin{abstract}
The Model of Friendly Health Services for Adolescents and Young People (SSAAJ for its acronym in Spanish) corresponds to a process created to guarantee that health institutions generate spaces and forms of comprehensive and differential care for the population between 10 and 29 years of age, allowing the Sexual Rights and Reproductive Rights (DSDR for its acronym in Spanish) according to the health needs and expectations that affect them. Objective: to investigate the knowledge, attitudes and experiences in DSDR in a group of adolescents and young people linked to the SSAAJ. Materials and methods: through a qualitative study with a phenomenological design, 13 semi-structured interviews were conducted with adolescents and young people between 13 and 21 years of age. Results: regarding knowledge about DSDR, male adolescents have some notions about human rights, but are unaware of the different DSDR that exist. In women there is more knowledge about DSDR. The attitudes of adolescents towards DSDR are favorable once they identify what they represent in the approach to sexuality. However, they consider that their experiences regarding the exercise of DSDR are limited for their enforceability and guarantee due to the lack of knowledge they have from them. Conclusions: it was found that the knowledge, attitudes and experiences of adolescents and young people about DSDR are influenced by family and cultural factors that impact their life trajectories.
\end{abstract}

\section{Key words}

Sexuality, reproductive rights, health services, adolescent, adolescent health services (Source: MeSH, NLM).

\section{Conhecimentos, experiêncios e atitudes sobre direitos sexuais e direitos reprodutivos em adolescentes e jovens}

\section{Resumo}

O Modelo dos Serviços de Saúde Amigáveis para Adolescentes e Jovens (SSAAJ) corresponde a um processo criado para garantir que as instituições de saúde gerem espaços e formas de atenção integral e diferencial para a população entre 10 e 29 anos, permitindo que se garantam os Direitos Sexuais e Direitos Reprodutivos (DSDR) de acordo com as necessidades e expectativas em saúde que os afetam. Objetivo: indagar os conhecimentos, atitudes e experiências em DSDR em um grupo de adolescentes e jovens vinculados aos SSAAJ. Materiais e métodos: mediante um estudo qualitativo com desenho fenomenológico se realizaram 13 entrevistas semiestruturadas aos adolescentes e jovens entre os 13 e 21 anos. Resultados: em conhecimentos perante os DSDR os adolescentes homens têm algumas noções sobre direitos humanos, mas desconhecem os diferentes DSDR que existem, nas mulheres predomina maior conhecimento sobre os DSDR. As atitudes dos adolescentes frente aos DSDR são favoráveis assim que identificam o que representam na abordagem da sexualidade. No entanto, consideram que suas experiências sobre o exercício dos DSDR se veem limitadas para sua exigibilidade e garantia pelo desconhecimento que têm deles. Conclusões: Encontrou-se que os conhecimentos, atitudes e experiências dos adolescentes e jovens sobre os DSDR estão influenciados por fatores familiares e culturais que impactam suas trajetórias de vida.

\section{Palovras chave}

Sexualidade, direitos sexuais e reprodutivos, serviços de saúde, adolescente, serviços de saúde do adolescente (Fonte: DeCS, BIREME). 


\section{Introducción}

La sexualidad es una dimensión esencial e inherente del ser humano, siendo un resultado de la interacción de factores psicológicos, biológicos, culturales, socioeconómicos, éticos y religiosos, plasmandose en todo lo que se hace o se piensa (1), de la cual se desglosan cuatro holones que se interrelacionan, siendo los siguientes: la reproductividad, el género, el erotismo y el vínculo afectivo (2). A lo largo del tiempo se han construido una serie de creencias y estándares sociales sobre la sexualidad, lo que conlleva a que esta sea concebida como un tabú para muchas poblaciones (3). Dichas representaciones afectan la vivencia y reconocimiento en la exigibilidad de los DSDR.

Por consiguiente, es importante reconocer que los DSDR están enmarcados dentro de los Derechos Humanos (DH), puesto que estos son definidos por las Naciones Unidas como: "derechos inherentes a todos los seres humanos, sin distinción alguna de raza, sexo, nacionalidad, origen étnico, lengua, religión o cualquier otra condición" (4, parr.1). Por tal motivo, de los DH se desglosan los DSDR, los cuales buscan salvaguardar la vida, la salud, la integridad y la autonomía de las personas (5). Es importante reconocer que los DSDR están encaminados a garantizar el ejercicio y desarrollo libre, informado, saludable y satisfactorio de la sexualidad, de una manera gradual y con base en el conocimiento científico, sin coacción y libre de toda forma de violencia, sin miedos, temores o prejuicios que limiten la expresión de estos derechos de acuerdo con lo estipulado por el Ministerio de Salud en Colombia. Los DSDR deben permitir el acceso y garantía a servicios de salud de calidad para su goce efectivo.

El ejercicio y exigibilidad de los DSDR impacta de forma directa la vivencia de la sexualidad en adolescentes y jóvenes, siendo éstos el $16 \%$ de la población en el continente americano (6). Adicionalmente, se evidencia mayor vulnerabilidad de los mismos en esta etapa del curso de vida, derivado de algunos procesos de socialización secundaria donde emergen necesidades de aprobación social y reconocimiento por parte de los pares, produciendo que adolescentes y jóvenes se encuentren inmersos en situaciones de mayor riesgo para su salud $(9,10)$, tales como: el consumo de sustancias psicoactivas, agresiones físicas y verbales, poco acceso al conocimiento sobre prácticas de autocuidado en la salud sexual y salud reproductiva (SSR); lo cual podría desencadenar en hechos como el embarazo en la adolescencia, presencia de infecciones de transmisión sexual (ITS) y procesos de aborto en lugares clandestinos.

Es importante tener presente que la adolescencia es un momento del desarrollo en el que existen múltiples cambios cognitivos, psicológicos, físicos, sexuales y sociales, mediados por las transformaciones hormonales y de maduración cerebral, lo que genera un incremento de la curiosidad por explorar el ejercicio de su sexualidad (11). En este mismo sentido, es relevante tener en cuenta las transformaciones que vive el adolescente a nivel psíquico, realizando procesos de simbolización, introyección, proyección y duelo $(11,12)$, lo que conlleva a un desequilibrio psíquico que genera situaciones vinculadas a crisis de identidad (12,13), así pues, Gómez (8) argumenta que, por esta razón, los adolescentes y jóvenes buscan una similitud e identificación en grupos sociales. Adicionalmente, el adolescente se ve inmerso en un mundo adulto, en el que se encuentra la narrativa de sus progenitores fuertemente influenciada por sus vivencias, generando una mayor angustia respecto a la vivencia de la corporalidad (37).

A su vez, a nivel mundial se reporta que los adolescentes inician su vida sexual en promedio antes de los 15 años (14), lo que conlleva la necesidad de abordar los DSDR. Especialmente, se requiere priorizar las prácticas de autocuidado en la salud sexual y salud reproductiva a nivel educativo (14), las investigaciones evidencian 
diferencias por género, siendo las mujeres las que tienen un nivel menor de educación en sexualidad que se refleja en las prácticas sexuales de riesgo.

Según cifras de la Organización Mundial de la Salud (15), 16 millones de adolescentes jóvenes entre 15 y 19 años de edad y 1 millón de niñas menores a 15 años dan a luz cada año, la mayoría de estas pertenecen a países de ingresos bajos y medios. En América Latina, un tercio de los embarazos ocurren en mujeres menores de 18 años, siendo casi el 20\% menores de 15 años, siendo las complicaciones en el embarazo y el parto la segunda causa de muerte en adolescentes. En cuanto al contexto colombiano, según la Encuesta Nacional sobre Demografía y Salud (EDNS) realizada en el año 2015 (16), el 17.4\% de las mujeres entre 15 y 19 años son madres o esperan su primer hijo. En cuanto a los procesos de interrupción del embarazo, más de 40 millones de abortos se realizan anualmente en el mundo y 19 millones son realizados con procedimientos inseguros, generando índices de mortalidad materna asociados a prácticas de riesgo frente al aborto (17), el cual en Colombia según el DANE (12), presenta una tasa alta de mortalidad infantil en madres adolescentes, con 25 defunciones por casa 1.000 nacimientos, siendo el proceso de aborto la causa principal. Adicionalmente, las prácticas de riesgo conllevan a procesos de mayor transmisión de VIH en población adolescente debido a que 2,1 millones de menores de 15 años a nivel mundial se encuentran diagnosticados con VIH, esto podría asociarse a que carecen de acceso a servicios de salud oportunos (18).

Otro de los DSDR que se encuentran presentes en las afectaciones a la población son la presencia de violencias de género, especialmente la vulneración del derecho a la vida, a la integridad física, psicológica y sexual de la mujer, siendo esta una de las principales causas de muerte en mujeres entre los 15 y 44 años de edad en el mundo, a causa de actos como: abusos sexuales, abusos físicos, violaciones, muertes por arma de fuego, lesiones por agresiones de la pareja y el suicidio (19). A nivel local, en la ciudad Santiago de Cali de acuerdo con el boletín epidemiológico del Observatorio de Salud Mental de la Secretaría de Salud Municipal de Cali, para el 2020 se notificaron 2.388 casos de violencia de género e intrafamiliar, entre los tipos de violencia que se reportan son la física, psicológica, sexual, económica o patrimonial. Las edades mayormente afectadas son: $35 \%$ en personas entre 29 y 59 años, un $25 \%$ en personas entre 18 y 28 años, un $17 \%$ en personas entre 12 y17 años y un $9 \%$ en personas entre 6 y 11 años.

Las problemáticas descritas anteriormente dan cuenta de las diversas vulneraciones frente a los DSDR por lo que se procede a describir cómo se presentan en la Comuna 18 de la ciudad de Cali, sobre la cual se desarrolló el presente estudio.

Según el informe "Cali, ¿cómo vamos en la niñez?” en el año 2016, la tasa de fecundidad de adolescentes en la comuna 18 que se encuentran en el rango de edad de los 15 y 19 años es de 45,77\%. Respecto a la mortalidad materna, se presentan 71,1 muertes de madres por cada 100.000 nacidos vivos. Adicionalmente, otro estudio evidencia que el porcentaje de violencia intrafamiliar es del 12\% en los diferentes sectores y el de violencia sexual equivale al $48 \%$, situando a la comuna 18 en Cali como una de las que más presenta agresiones sexuales dirigidas a poblaciones vulnerables (20). Por otro lado, se han realizado estudios sobre el uso y cumplimientos de dichos derechos a nivel latinoamericano y nacional, encontrando que en Latinoamérica hay muchos temas a trabajar con adolescentes frente a los DSDR. Uno de éstos es el tabú y las creencias que tienen las familias frente a la sexualidad, que impide que los jóvenes se acerquen a los puestos de salud para informarse sobre la sexualidad y la reproductividad por temor a que los padres o familiares se enteren $(21,22)$, lo que da indicios de que algunos adolescentes y jóvenes no conocen el derecho a la confidencialidad. Este hecho genera consecuencias como las prácticas sexuales de riesgo $(23,24,25)$. 
En este sentido, un estudio realizado por Pacheco-Sánchez et al. (26) en la ciudad de Bogotá encontró marcadas diferencias de género entre los adolescentes. Las mujeres reportan un discurso negativo frente a la sexualidad, como que el noviazgo es malo, no se pueden tener relaciones sexuales porque es algo peligroso, buscando con ello que no se relacionen con el sexo opuesto ya que pueden quedar en embarazo; mientras que los varones tienen mayor libertad para hablar de sexualidad en la familia, con una postura marcada desde el lado del placer y de la prevención de alguna ITS, concibiendo al joven una mayor aprobación de iniciar la vida sexual, pero con cuidado y protección. Los jóvenes tenían una concepción de que las mujeres son las responsables de las consecuencias de las relaciones sexuales (embarazo), mientras que los hombres son vulnerables frente a éstas y por tanto deben protegerse.

En Colombia, según los estudios encontrados, se presenta desinformación de los DSDR por parte de los adolescentes y jóvenes, lo cual evidencia la necesidad de abordar el derecho a la información, la libre expresión y el conocimiento de los métodos de planificación $(7,27,29)$, especialmente, se requiere mejor acceso a los servicios de salud y campañas orientadas a promover la educación sexual en entornos cercanos a la población como las instituciones educativas y la comunidad. Algunos factores que se relacionan con la información de calidad en derechos sexuales y derechos reproductivos son: (1) educación familiar en el tema (2) desigualdades de género (3) mitos y prejuicios sobre la vivencia de la sexualidad, así como diferentes determinantes sociales de la salud $(26,27,28)$.

En cuanto a las investigaciones realizadas en la ciudad de Cali se encuentra que Valencia, et al. (30) abordó las experiencias, expectativas y percepciones que tienen los adolescentes frente a los servicios de salud amigables para adolescentes y jóvenes (SSAAJ); encontró que hay carencia de espacios acordes, horarios para la atención y falta de personal, como también de capacitación por parte del mismo, lo que genera que el adolecente asista cuando ya está el problema.

La presente propuesta evidencia la necesidad de abordar los DSDR teniendo en cuenta la legislación colombiana, la cual ha desarrollado diversas normatividades que permiten el ejercicio, protección, exigibilidad y exigencia de los DSDR en niños, niñas y adolescentes, como la Comisión Nacional Intersectorial para la Promoción y Garantía de los Derechos Sexuales y Reproductivos con el fin de que se implementen planes y acciones requeridas para el cumplimiento de las políticas y la garantía de los DSDR (31).

A partir de lo anteriormente expuesto y la importancia de trabajar con adolescentes y jóvenes respecto al tema, la presente investigación tuvo como objetivo indagar las concepciones, experiencias y actitudes sobre derechos sexuales y derechos reproductivos en un grupo de adolescentes de la comuna 18 de la ciudad de Cali, Colombia. Los conocimientos, son entendidos como toda la información sobre qué son los DSDR y las diversas prácticas de riesgo vinculadas a su exigibilidad y vulnerabilidad (40). Las actitudes se refieren a todas aquellas posiciones favorables o desfavorables frente al ejercicio y exigibilidad de los DSDR (41). Finalmente, las experiencias son una construcción personal, social e histórica que se da a partir de las vivencias frente a situaciones de la SSR o los DSDR (42).

\section{Método}

Se realizó una investigación cualitativa, con diseño fenomenológico (32), orientada a indagar los conocimientos, actitudes y experiencias vinculadas desde las subjetividades de los participantes, partiendo de sus trayectorias personales en el ejercicio y exigibilidad de los DSDR. El presente estudio se articula al proyecto Macro sobre el Análisis de los Servicios de Salud 
Amigables para Adolescentes y Jóvenes en 31 instituciones de salud. Para el presente estudio se focaliza la participación en una de las zonas del proyecto macro. En este caso participaron 13 adolescentes y jóvenes con edades de 13 y 21 años, los cuales fueron seleccionados a través de un muestreo por conveniencia (33), partiendo de aquellos adolescentes y jóvenes que alguna vez han participado en actividades ubicadas en el centro de salud de la comuna 18 en la zona Ladera de la ciudad de Cali. Del total de participantes, se logró la vinculación de siete hombres y seis mujeres.

La técnica de recolección de información fue la entrevista semiestructurada (34) que duró 45 a 60 minutos por participante, la entrevista permite construir una guía inicial de preguntas asociadas a las categorías de análisis construidas previamente por las investigadoras, en este caso, derivadas a partir de la revisión de literatura sobre antecedentes. Las principales categorías abordadas fueron: conocimientos en DSDR, actitudes frente a los DSDR y experiencias de ejercicio y exigibilidad de los DSDR. Durante el proceso de análisis emergió la categoría participación familiar en la vivencia de los DSDR, la cual hace referencia a todas aquellas acciones presentes en los procesos de las dinámicas familiares que influyen en los imaginarios sobre derechos sexuales y derechos reproductivos en la población participante.

El procedimiento para la recolección de la información se realizó a partir de la base de datos de profesionales de la salud vinculados a los Servicios de Salud Amigables de la ciudad de Cali en el proyecto macro. Los criterios de inclusión para la selección fueron: adolescentes entre los 13 y 21 años, escolarizados y que asistieran a los SSAAJ. Posteriormente, se solicitó a los acudientes de los menores de edad y a los adolescentes y jóvenes la firma del consentimiento y asentimiento informado. Es importante aclarar que los nombres utilizados en el análisis de la información pseudónimos. Adicionalmente, se realizaron entrevistas semiestructuradas para la recolección de datos, las cuales se efectuaron en un lugar en el que los adolescentes y jóvenes se sintieran cómodos para expresar sus conocimientos, actitudes y experiencias. Finalmente, se procedió al análisis de la información utilizando la sistematización de los datos cualitativos mediante el programa Atlas Ti V. 8.0 y se realizó el análisis de tipo temático (35), organizando las verbalizaciones y la segmentación de las narrativas por ejes temáticos centrales de las categorías realizadas previamente desde la sistematización de las voces de los participantes en aspectos que tenían congruencias y discrepancias.

Igualmente, se tuvo en cuenta que el estudio se realizó con seres humanos y por tanto se rige bajo la resolución 008430 (36) que dirige la normatividad para llevar a cabo investigaciones en salud. Igualmente, la ley 1090 (37) que reglamenta el ejercicio profesional de la psicología. En este mismo sentido, el proyecto macro obtuvo el aval ético institucional para su implementación, y se incluye la aplicación del consentimiento y asentimiento informado por parte de los participantes vinculados al proceso de las entrevistas.

\section{Resultodos}

A continuación, se expondrán los datos obtenidos en las entrevistas que fueron analizados a partir de las categorías de análisis las cuales fueron: conocimientos frente a los DSDR, actitudes frente a los DSDR, experiencias asociadas a los DSDR y participación familiar en la vivencia de la sexualidad.

\section{Conocimientos frente a los DSDR}

Esta categoría buscó identificar las ideas o nociones que tenían los adolescentes y jóvenes adolescentes sobre los Derechos Humanos y los Derechos Sexuales y Derechos Reproductivos, encontrando que los adolescentes y jóvenes describen algunos DH como: el derecho a la vida, a la educación, a la salud y a la igualdad. Así mismo, reconocieron 
a la educación primaria como la primera fuente de información sobre este grupo de derechos. Respecto a la relación entre DH y conocimientos sobre DSDR, los participantes no logran vincular que se desprenden de éstos y su vínculo directo con los propósitos para la garantía, exigibilidad y ordenamiento jurídico. Desde la perspectiva de los hombres, se encontró que algunos de ellos hablaron sobre los DSDR sin necesidad de apoyo, teniendo muy presente el derecho a tener relaciones sexuales consensuadas, anclándolo a la libertad que tienen todas las personas como derecho fundamental: "El que más recuerdo, es respetar la opinión de la persona, la postura, el derecho a tener relaciones coitales, si la otra persona pues no está de acuerdo, tenemos que respetar su palabra, no obligar a hacerlo (...)" (Joher, 16 años).

En cambio, en el discurso de algunas mujeres se evidenció el reconocimiento de varios DSDR, sobre todo desde el cumplimiento de los mismos en situaciones de vulneración por toma de decisión frente a la orientación sexual, si ser madre o padre, querer iniciar la vida sexual, tener relaciones sexuales consensuadas y escoger la pareja.

Pues si digamos yo llego a quedar embarazada no, y a mí me están diciendo usted no puede tener al niño, si yo quiero y asumo la responsabilidad lo puedo tener, porque es un derecho...pues por ejemplo cuando hay violación, la persona debe hablar, cuando hay un embarazo y le están negando tener el niño, también debe hablar, o cuando las obligan a prostituirse que les dicen usted tiene que estar con tal persona, ellas tienen el derecho con quien tener relaciones sexuales. (Mónica, 13 años)

Es importante acotar que una de las adolescentes entrevistadas atribuyó el desconocimiento de los DSDR a la no garantía de los mismos, señalando que este hecho genera que se produzca una vulneración debido a que la misma población no es consciente de lo que puede ejercer, permitiendo que otras instituciones o agentes no realicen el cumplimiento de dichos derechos.

Frente a los conocimientos sobre rutas de atención, en caso de vulneración de derechos, se encuentra que los adolescentes no identifican las instancias y el paso a seguir para activar una ruta de atención, sin embargo, logran identificar organizaciones competentes que están involucradas en el proceso como lo son: la Fiscalía, la Policía, la IPS o llamar a una línea de atención. En cuanto al testimonio de una de las participantes se evidencia que hubo maltrato psicológico por de parte de su pareja hacía ella, afirmando que no había caído en cuenta de activar una ruta de atención, puesto que no vió la importancia, considerando que la situación que evidenció ocurría normalmente en un contexto de relación de pareja. Por tanto, varios adolescentes veían este proceso como necesario sólo en caso de agresión física o sexual. "Cuando uno a veces tiene problemas con la pareja y empieza a tratar mal vocalmente...No sé...Tal vez, yo creo que eso...No sé, nunca lo pensé así, solamente no fui" (Carmen 18 años).

Por lo tanto, se puede decir que los adolescentes, frente a los conocimientos en DSDR, tienen saberes a través de las experiencias que han vivenciado en su historia de vida o las situaciones que han escuchado en agentes cercanos a ellos, como las instituciones educativas o miembros de su familia. En cuanto al acceso o garantía de los DSDR, no se encuentran diferencias entre hombres y mujeres, puesto que en ambos se evidenció acceso a esta información por parte de los agentes anteriormente mencionados.

\section{Actitudes frente a los DSDR}

Dicha categoría buscó revisar las posturas, opiniones y posiciones que tienen los adolescentes sobre los DSDR a partir de las experiencias y vivencias que han tenido. Se encontró que la mayoría de los participantes tienen una postura favorable hacia los DSDR, notándose que se 
le atribuye por parte de ellos una percepción de importancia y beneficio a través del ejercicio de estos derechos, mostrando un interés en indagar sobre ellos para poder enriquecer la vivencia de su sexualidad. "Primero que todo que es indispensable conocerlos y saber de ellos, porque cuando uno no sabe, uno no sabe lo que se está vulnerando, porque hay muchas personas que no cumplen con ellos, hasta las mismas instituciones educativas no cumplen" (Elena, 17 años).

Asimismo, se encontró una necesidad por parte de los adolescentes de hacer más énfasis en la educación y la divulgación de los DSDR, puesto que ellos mismos expresan querer aprender más sobre este tema, con el fin de saber cómo proceder en caso de vulneración. “(...) yo diría que tendría que haber un espacio entre semana, no todos los días, pero al menos, un espacio por día, donde a uno le puedan hablar sobre eso, y cómo usar todos los métodos" (Andrés, 18 años).

Adicionalmente, se encontraron posturas desfavorables por parte de dos de los participantes que están ligadas a una resistencia frente al ejercicio de los DSDR, que se debe a las vivencias, creencias y proyecto de vida de cada uno de ellos. “(...) pues el de la vida (...) acuden al aborto (...) no estoy de acuerdo en absoluto, porque son vidas" (Maira, 17 años). "Creo que por qué no me interesa los DSDR... porque mi mentalidad es ser sacerdote (...) siempre lo he querido ser" (José, 14 años).

\section{Experiencias asociadas a los DSDR}

En esta categoría se indagaron las vivencias que cada participante ha construido en sus trayectorias de vida en el ejercicio de los DSDR a partir de lo percibido en las experiencias consigo mismo y otras personas de sus escenarios de socialización. A partir de lo anterior, se evidenciaron situaciones de vulneración de derechos, sobre todo las que tienen que ver con la interrupción voluntaria del embarazo (IVE), puesto que la mujer accedió

a un método que coloca en riesgo su vida, vulnerando el derecho a tener acceso a los métodos anticonceptivos, seguros y eficaces, y a acceder a servicios de salud y atención médica que garanticen una maternidad segura. Así mismo, se encuentra la familia como mediador en el acompañamiento del acceso de los DSDR. Cabe resaltar que dichas situaciones son experiencias relatadas de amigos de los entrevistados y estos no acudieron a rutas de atención.

\begin{abstract}
Pues una amiga una vez me contó que ella no quería tener el hijo y que ella se había comprado una pasta para eso y que el niño se le había venido... Pues, ella me contó, que ella había ido por el centro y la había comprado...ella sangraba mucho y le venían partecitas y que la llevaron al médico por lo que estaba sangrando demasiado... Si y pues ella estaba sangrando demasiado y los médicos le dijeron que era aborto y ella negaba que lo había abortado...Si, pues ellos (los papás) sabían que estaba embarazada, pero ellos no la apoyaban, pues yo creo que por eso ella abortó. (Mónica, 13 años)
\end{abstract}

Igualmente, se encuentra que los adolescentes reconocen la importancia del derecho que tienen de recibir información sobre sexualidad, sin embargo, afirman que se les dificulta dirigirse hasta los servicios amigables de la zona por la distancia, la dificultad en el pago de transporte y la alimentación durante el tiempo en el que se encuentran en la institución. Sin embargo, en el discurso de los participantes se evidenció que los adolescentes sienten que los servicios amigables brindan un buen servicio, puesto que ofrecen los servicios a cualquier joven, sin discriminar sexo o si se tiene afiliación médica. Así mismo generan una atención oportuna, siendo muy claros en el lenguaje que manejan para dar la información a los adolescentes, como también guiándolos en el proceso que deben realizar. Todo esto bajo la confianza que sienten los adolescentes de conversar sobre temas de sexualidad sin miedo a prejuicios. 
En cuanto a las rutas de atención se encuentra que los adolescentes, al no haber hecho uso de éstas, el conocimiento y empleo de una ruta de atención es prácticamente un terreno sin explorar en la trayectoria de vida de los adolescentes entrevistados. Frente a las experiencias asociadas al cumplimiento de DSDR, los adolescentes expresaron situaciones en las que hicieron uso de sus DSDR, como lo fue: escoger a su pareja, decidir si se quiere iniciar su vida sexual o no, el compañero/a sexual, respetar la orientación sexual, acceder a información veraz y científica, a escoger el método anticonceptivo, cuántos hijos se desea tener y en qué momento.

Se encontraron diferencias de género respecto al acceso de información veraz y científica sobre el tema de DSDR, puesto que uno de los participantes afirma que cuando tenía curiosidad sobre el tema de la sexualidad sus padres le ayudaron a acceder a los servicios amigables para despejar las dudas que tenía, mientras que a otra participante le costó mayor tiempo conversar sobre el tema con su madre, ya que esta decía que aún no era la edad adecuada para hablarlo. Por tanto, al momento de indagar por garantías que les permitan un ejercicio saludable y libre de su sexualidad, se evidenció que el componente familiar se hace presente al momento en que decide compartir abiertamente la información con un adolescente hombre que con una mujer.

Cuando yo tenía la idea de iniciar mi vida sexual, mis padres se sentaron conmigo y pues buscaron las alternativas para evitar cualquier error, yo pienso que ahí se valieron, porque no se negaron, buscaron los medios para que me llegara la información. (Joher, 16 años)

Respecto a las vivencias y las percepciones de los adolescentes frente a situaciones de vulneración de sus DSDR. Se encontró principalmente que la familia, en gran parte de los casos, se presenta como el sistema principal que está vinculado al cumplimiento o no de los DSDR, puesto que en la mayoría de los casos se oponen al deseo del joven de vivir su sexualidad, a través de la búsqueda de métodos de protección, acceso a servicios médicos o información. Por lo que, en situaciones como estas, se evidencia cómo la historia y la trayectoria familiar influyen en la vivencia de los adolescentes frente al ámbito de su sexualidad, como se evidencia en el siguiente fragmento:

Cuando quedan embarazadas o algo, y los papás le dicen, no usted no va a tener el niño, porque la gente va a mal pensar, que la gente va a hablar. Esas personas, como le digo, dicen no, yo quiero ser mamá, yo quiero sacar mi hijo adelante, ellas tienen que tomar la decisión y si ya la tomen ya nadie les puede decir que no. (Mónica, 13 años)

Se encontró que una de las barreras para asistir a los Servicios Amigables es al momento de atender a un adolescente, en casos donde el beneficiario es menor a los 13 años, ya que requiere del acompañamiento de un adulto para participar en el programa, lo que limita en diversas ocasiones la participación de los jóvenes en las actividades brindadas.

\section{Participación familiar en la vivencia de la sexualidad}

Como se ha observado en los resultados anteriormente expuestos, la familia, emerge como un componente fundamental en la vivencia de la sexualidad para los adolescentes, por lo que se encontró la necesidad de dar lugar a este sistema, como esfera influyente en las construcciones que los adolescentes han realizado sobre los DSDR, además de las instituciones educativas donde los adolescentes constituyen sus posturas frente a la realidad.

En primer lugar, algunos adolescentes expresaban su visión de la familia como sistema de apoyo frente a las curiosidades que surgen en este momento de la adolescencia, propiciando información sobre la 
sexualidad, ya sea por medio de conversaciones o buscando ayudas externas, con el fin de que los jóvenes reciban el asesoramiento completo sobre las dudas que tengan, como lo son los servicios amigables que se brindan en la IPS del sector. En este mismo sentido, algunos de los cuidadores primarios de los jóvenes prefieren que ellos consulten esta información autónomamente $\mathrm{o}$ brindan indicaciones breves sobre lo que deberían realizar en sus experiencias sexuales, generando que el adolescente acuda a pares o agentes externos para aclarar estas dudas: "Muy poco la verdad. Nada, es que ese tema uno lo habla más como con los amigos, que los papás mantienen en la casa. Entonces con los papas uno casi no" (Juana, 17 años).

Por otro lado, se evidenció una diferencia entre el diálogo que se establece con los adolescentes hombres y con las mujeres. Los hombres entrevistados expresaban que sus acudientes prefieren reservar o frenar las conversaciones sobre temas de sexualidad, limitándolo a consejos o sugerencias precisas frente a los riesgos o consecuencias que podrían tener si no tiene precaución con sus acciones:

Pues mi papá no vive con nosotros, pero cuando a veces, me ha preguntado si pues ya, viene me dice que me cuide, que use condón. Obviamente ellos saben, sino que yo por lo menos con ellos casi no he hablado con ellos. (Yordy, 15 años)

Mientras que los padres o cuidadores de las mujeres encaminan el discurso a advertir a la mujer de no desarrollar una maternidad prematura, previniendo además de cualquier tipo de agresión o abuso por parte de la pareja que se establezca.

Mi papa es muy machista (...) Yo tengo un hermano y mi hermano, hace y deshace y él quiere que yo no haga y deshaga, entonces él siempre me ha dicho, si usted la caga, se larga. Entonces yo siempre le digo, listo, si la cago me voy, pero si mi hermano la caga también se va, entonces hay mismo revira y dice, pero porque es que él es hombre y también se tiene que meter la cabeza a responder por el muchachito, pero él dice que no. (Daniela, 21 años)

Por otro lado, la religión emerge como un factor que influye en la construcción de percepciones frente a la vivencia de sexualidad, desarrollándose límites o prohibiciones frente a la experiencia en sexualidad.

Hay algunas situaciones de familias que se han visto, de que, no lo dejan elegir, si no que le toca...una persona muy religiosa le prohíbe a su hija, y por prohibirle, su hija hace cosas que no debe, o sea, comienza su relación sexual muy pronto, cosas así, son algo que me pertenece a mí, que yo decido y no que otra persona decida por mí. (Maira, 17 años)

En los relatos de los participantes se destaca el énfasis que hacen los padres en los diálogos que sostienen con sus hijos, en donde expresan las vivencias de su sexualidad en la adolescencia y manifiestan el deseo de que sus hijos no repitan las acciones realizadas, evitando cometer errores, como lo califican los padres. Así se evidencia en el siguiente fragmento:

Nada. Pues digámoslo así, como cuídese porque ella quedó en embarazo de mí muy joven, que no quiero que le pase lo mismo que a mí (...) Entonces es como un temor no, porque yo digo que ah no puedo hacer esto porque qué tal que pase lo mismo. Entonces uno queda como amarrado a lo que le dicen. (Juana, 17 años)

\section{Discusión}

A partir de los resultados encontrados en el presente estudio, se reconocen diversos factores que se ven involucrados en la apropiación de los DSDR. Lo 
anterior se vincula con la propuesta del Modelo de Determinantes Sociales de la Salud en el cual las circunstancias del contexto en que las personas habitan se encuentran en permanente interacción con el sistema familiar, económico, político, comunitario, cultural, educativo y de salud, lo que conlleva a generar algunas aproximaciones frente a las decisiones orientadas a la salud sexual y salud reproductiva de adolescentes y jóvenes. Algunos estudios como los realizados por Pacheco-Sánchez et al. (26), Vinaccia et al. (29) y González-González (27), evidencian las barreras asociadas al género sobre la apropiación de los DSDR y la formación en sexualidad, similar a lo hallazgos evidenciados en este estudio. Esto se debe a elementos como: las creencias familiares, la religión y los estigmas sociales, dificultando la garantía de los DSDR para las mujeres, mientras que el hombre tiene mayor libertad para expresar, vivir y disfrutar de su sexualidad. En las narrativas de los participantes se resalta una marcada concepción respecto al tema, en el que se vincula a la mujer directamente con la maternidad, siendo concebida como una limitación para la realización del proyecto de vida, mientras que al hombre se le permite una vivencia de la sexualidad a través del goce corporal, admitiéndolo como algo normal e implícito en su interrelación con los otros.

Por otro lado, en el discurso de los participantes se percibe constantemente la figura de la familia como un sistema fundamental que influye en la vivencia de la sexualidad en el adolescente. Así pues, a través de la narrativa de adolescentes y jóvenes, la familia emerge como el entramado de significados, creencias y tradiciones que recibe a los adolescentes en el medio social y en la mayoría de los casos, imparte dichas concepciones y las convierte en una demanda para el adolescente, promoviendo una introyección psíquica y emocional en éste, lo que genera que la vivencia de sexualidad se vea permeada, desembocando en el síntoma que, según Lacan (9), se observa a través de los actos que reivindica su capacidad de ser un ser sexuado, como es el embarazo en la adolescencia, elementos que se registraron en las experiencias narradas a través de los discursos.

En cuanto al sistema familiar, se logra vislumbrar en los adolescentes la estructuración simbólica que compone el discurso de los padres, en el que se articulan distintos registros simbólicos a nivel moral y a nivel médico, de modo que los diálogos entre el adulto y el adolescente se centran principalmente en el control y la restricción sobre su cuerpo, más que en el goce y el disfrute que genera el descubrimiento de la sexualidad; este aspecto es similar a lo que afirma Jones (38) acerca de los diferentes discursos que coexisten en la sexualidad del adolescente. Es por esto que Ramos (21) afirma que aún se encuentran sociedades tradicionalistas en la que aparecen los diversos tabúes e imaginarios que impiden hablar de sexualidad de una forma natural, obstaculizando el acceso a información sobre los DSDR, sobre todo a la población adolescente.

Por otro lado, en algunos relatos se encontró una actitud desfavorable frente a los DSDR desde la perspectiva de los adolescentes, lo que se explica en algunos casos desde el poco reconocimiento que ellos reciben por parte de las instituciones, en el que no es nombrado como un ser en transición, puesto que es entendido en mayor medida solo desde sus dinámicas reproductivas biológicas y funcionales; en consecuencia, se excluyen las representaciones simbólicas que éste tiene del mundo, además del deseo que en él habita, produciendo que se conciba al adolescente desde los juicios sociales y desde los estereotipos que el mundo adulto posee, lo anterior represente un desafío en la formación de los profesionales que acompañar en los procesos de intervención durante esta etapa del ciclo vital.

Desde la perspectiva de adolescentes y jóvenes, en el discurso de los padres se evidencian diversas preocupaciones manifestadas frente a la transmisión intergeneracional de sus experiencias de vida a la formación de los hijos, expresando mayores temores frente a la repetición de 
situaciones que afectaron sus proyectos de vida en el abordaje de la sexualidad. Es por esto que en el discurso de los participantes se logra validar los planteamientos de Orcasita, Cuenca, Montenegro, Garrido y Harderlein (39), respecto a la repetición en los padres de familia de aquellos simbolismos transmitidos por sus progenitores en la adolescencia, en los que se evitaba hablar de temáticas relacionadas con la sexualidad, evidenciándose de forma más específica en los padres que en las madres, puesto que el encuentro sexual es visto como una vía directa al proceso de embarazo o la transmisión de una ITS, lo que infunda a nivel psíquico miedo y angustia respecto al encuentro con los otros que el adolescente pueda llevar a cabo.

Otro elemento que dificulta el acceso a la información por parte de la población es las condiciciones socioeconómicas de la comuna 18 , como lo caracterizó Osorio y Aguado (20) y lo expresaron los participantes. Dicho nivel afecta los procesos de transporte y alimentación de los adolescentes y jóvenes que asisten a los Servicios de Salud Amigables. A su vez, se debe sumar la accesibilidad que hay entre las viviendas del sector y la entidad de salud, haciendo que para algunos jóvenes se conviertan en barreras para asistir a las citas y procesos de formación. Es por esto que se considera pertinente diseñar, planear y ejecutar intervencionesgrupalesqueinvolucrenalas familias o cuidadores primarios de estos adolescentes con el propósito de fomentar un rol más activo dentro de la comunidad, siendo éstos los multiplicadores principales del sector. Adicionalmente, en cuanto a las fuentes de información, se encuentra que los servicios amigables son el primordial recurso al que los adolescentes acceden para conocer sobre sexualidad, elemento que, según Díaz et al. (7) presenta un desafío, especialmente se requiere potenciar campañas para acercarse a los jóvenes de forma que promueva en esta comunidad confianza o seguridad para obtener información por parte de la institución.
En la presente investigación se encontró que los Servicios de Salud Amigables de la comuna 18 realizan diversos eventos en lugares concurridos por la comunidad, lo que ha permitido mayor visibilidad para la promoción y garantía de los DSDR para adolescentes y jóvenes, como también la inclusión de procesos formativos en algunos usuarios que asisten con el fin de que sean jóvenes multiplicadores y de esta manera se conviertan en agentes de cambio para otros adolescentes y jóvenes de su entorno comunitario y educativo. Adicionalmente, los 13 participantes mencionaron satisfacción frente al uso de los servicios amigables en su comuna durante los procesos de formación que han recibido, especialmente por las metodológias participativas e interactivas que incluyen las actividades. Los participantes valoran que se diseñen acorde a sus necesidades y experiencias de vida. Sin embargo, este estudio recomienda seguir potenciando estas estrategias, especialmente campañas de difusión de información, que contengan un mensaje claro y directo para la población a la cual se dirigen los SSAAJ, permitiendo una cobertura más amplia del sector.

En cuanto al colegio, esta instancia puede considerarse como la segunda fuente de información como recurso de formación para los adolescentes a partir de lo expresado por los participantes. Igualmente, se evidencia una necesidad de implementar materias en los colegios en las que se hable exclusivamente de sexualidad; es decir, no solo desde el campo de la biología en áreas como ciencias naturales, si no que abarque la sexualidad de forma transversal en los contenidos propuestos por Rubio (2). Por lo tanto, lo que afirma Díaz, et al. (7) concuerda con lo expresado en los resultados de las entrevistas realizadas, frente a la necesidad de tener educación integral en sexualidad en los contextos educativos. Lo cual implica potenciar la formación de profesionales idóneos para los procesos de capacitación con adolescentes y jóvenes frente a las competencias que propone el Modelo SSAAJ en el acompañamiento integral. 
Adicionalmente, se requiere considerar la vinculación que los adolescentes perciben con las instituciones que realizan abordajes en DSDR . En muchos casos, dichos agentes de formación son considerados lejanos y ajenos por parte de los adolescentes a su realidad, puesto que quienes realizan las intervenciones se convierten en representantes de una norma adulta y no de un guía en salud, desde la experiencia y la comprensión. Es por esto que se debe buscar promover espacios de interacción y reflexión con los adolescentes que fortalezcan el vínculo entre la institución y el joven, en los que la relación se forje desde la horizontalidad y la aceptación del otro. Igualmente, los adolescentes han incorporado a su vida distintas dinámicas de relación que no solamente se infunde desde la interacción física, sino que aparecen factores digitales y mediáticos que trazan un modo de relación del adolescente con el medio exterior.

\section{Conclusiones}

Según los aspectos anteriormente abordados se concluye que las concepciones que tienen los adolescentes sobre DSDR se encuentran permeadas por diversos factores como lo son: las creencias familiares, religiosas e imaginarios sociales y culturales, elementos que generan en el adolescente una apropiación diferencial de los DSDR. Así mismo, el colegio y los servicios amigables son las principales instituciones en la que los adolescentes reciben conocimientos sobre sexualidad desde un enfoque de género y de derechos, que permite aproximarse al abordaje de los DSDR.

Respectoaladimensiónfamiliar, elprincipalanálisis se centra en las introyecciones y proyecciones que el adolescente ha realizado a través de su historia, de las cuales construye unos ideales del YO que se retroalimentan constantemente con las identificaciones que se realizan de los pares y la mirada del otro. Por consiguiente, este conjunto de modos de vinculación afectiva con los demás influye en la experiencia, vivencia, deseo y curiosidad por conocer acerca de la dimensión sexual y su evidencia en el ejercicio de los DSDR.

Todo lo mencionado anteriormente permite concluir que los conocimientos, experiencias y actitudes que tienen los adolescentes de la comuna 18 de Cali sobre los DSDR se encuentran mediados por la historia y la trayectoria de vida de cada individuo, como también por las normas familiares y el discurso de una sociedad tradicionalista que aún concibe la sexualidad como un tabú, generando barreras para el acceso a la información. Lo anterior lleva a que los jóvenes y adolescentes sientan discrepancias frente a la realidad que les acontece, a nivel físico y psíquico, puesto que en la mayoría de los casos no encuentran respuesta por parte de familiares u otras instituciones, debido al desconocimiento frente a la importancia de una educación integral en sexualidad que promueva la confianza frente a los diálogos que se establecen en el reconocimiento y exigibilidad de los DSDR.

Por tal motivo, los adolescentes y jóvenes se ven en la tarea de acudir a los pares para solventar la incertidumbre, en los cuales se gesta un vínculo de confianza y horizontalidad, características que deben promoverse en los espacios de acercamiento a las comunidades en diferentes sistemas que abarcan el ámbito familiar, educativo y de salud.

Finalmente, se plantea como recomendación para intervenciones futuras: el uso de estrategias educativas multimediales que generen un acercamiento del adolescente por distintos canales de comunicación, en el que no solo se geste un interés del adolescente en la temática de derechos, sino que también tenga la oportunidad de ser quien aborde un proceso autónomo de aprendizaje a través de estas plataformas y seguir promoviendo los SSAAJ como estrategia transversal para la apropiación de los DSDR.

Conflicto de interés: los autores declaran no tener conflictos de interés con la publicación de este artículo. 
Fuentes de financiación: Esta investigación estuvo articulada al proyecto de investigación "Análisis del Modelo de los Servicios de Salud Amigables para Adolescentes y Jóvenes (SSAAJ) para la promoción y garantía de los derechos sexuales y derechos reproductivos en la ciudad de Cali" (Código: 020100646), avalado por la Facultad de Humanidades y Ciencias Sociales y financiado por la Oficina de Investigación y Desarrollo de la Pontificia Universidad Javeriana Seccional Cali. 


\section{Referencios bibliográficos}

1. Arango I. Sexualidad humana. México D.F: Editorial El Manual Moderno; 2008

2. Rubio AE. Sobre la sexualidad humana: los cuatro holones sexuales [Internet]. México; 1996 [consultado My. 2019]. Disponible en: http:/www.dgespe.sep.gob.mx/public/genero/PDF/ LECTURAS/S_01_06_Sobre\%201a\%20sexualidad\%20humana.pdf

3. González-Vélez A, Monsalve V. Case study on Colombia: judicial standards on abortion to advance the agenda of the cairo programme of action. SUR - International Journal on Human Rights. 2013; 10(9): 1-26.

4. Naciones Unidas. Derechos Humanos [Internet]. [Consultado 3 May 2019]. Disponible en: http:// www.un.org/es/sections/issues-depth/human-rights/index.html

5. Dalén A, Guzmán D E, Molano P. La regulación de la interrupción voluntaria del embarazo en Colombia [Internet]. Bogotá: Centro de Estudios de Derecho, Justicia y Sociedad, Dejusticia; 2013 [Consultado 3 May 2019]. Disponible en: http://www.dejusticia.org/files/r2_actividades_recursos/ fi_name_recurso.339.pdf

6. Organización Panamericana de la Salud [Internet]. [Consultado 12 Oct 2020]. Disponible en: https:// www.paho.org/informe-salud-adolescente-2018/part-one-a-profile-of-adolescents-and-youth-inthe-americas.html

7. Díaz L, Torrente M, Gómez E. Perfil epidemiológico de la salud sexual y reproductiva de un grupo de adolescentes escolarizados: una perspectiva desde los derechos. Bello (Colombia). Medunab. 2011; 14(1): 15-25.

8. Gómez E. Adolescencia y familia: revisión de la relación y la comunicación como factores de riesgo o protección. Rev Inter Psico y Edu. 2008; 10(2): 105-122.

9. Valenzuela E, Casas L. Derechos sexuales y reproductivos: confidencialidad y VIH/SIDA en adolescentes chilenos. Acta bioethica [Internet]. 2007 [Consultado 3 May 2019]; 13(2): 201-215. Disponible en: https://scielo.conicyt.cl/scielo.php?script=sci_abstract\&pid=S1726-569X20070002 00008\&lng=en\&nrm=iso\&tlng $=$ es

10. UNFPA, Ministerio de Salud y Protección social, UNICEF. (2013). Consultoría para la evaluación de los Servicios Amigables de Salud para Adolescentes y Jóvenes en Colombia.Recuperado el 10 de abril de 2018.

11. Lacan, J. O simbólico, o imaginário e o real. Lugar de publicación: Argentina Editorial; Paidos 1953

12. Aberastury de Pichón-Rivière A, Knobel M, Dornbush A. La adolescencia normal: Un enfoque psicoanalítico [Biblioteca del educador contemporáneo, 127]. Buenos Aires: Paidós; 1971

13. Erikson, E. Observaciones sobre la identidad más general. México: Fondo de Cultura Económica; 1994. En Un modo de ver las cosas; p. 447-451.

14. UNICEF. Las niñas el VIH/SIDA y la educación. S.f. [Consultado 21 ag. 2017]. Disponible en: https://www.unicef.org/spanish/publications/files/Girls_HIV_AIDS_and_Education_(Spanish).pdf

15. Organización Mundial de la Salud. El embarazo en la adolescencia [Internet]. 2018 [Consultado 3 my. 2019]. Disponible en: https:/www.who.int/es/news-room/fact-sheets/detail/adolescentpregnancy

16. Profamilia. Encuesta Nacional de Demografía y Salud (EDNS) [Internet]. 2015 [Consultado 3 my. 2019]. Disponible en: https://profamilia.org.co/investigaciones/ends/

17. Amneris SU, Reyes EG, Figueroa MM, Batán Y. Comportamiento sexual y aborto provocado en adolescentes y jóvenes de escuelas de educación superior. Rev Cub Obste Y Gineco [Internet]. 2012 [Consultado 3 my. 2019]; 38(4). Disponible en: http://www.bvs.sld.cu/revistas/gin/vol38_4_12/ 
gin12412.htm

18. Organización Mundial de la salud. Infecciones de transmisión sexual [Internet]. 2016 [Consultado 21 ag. 2017]. Disponible en: http://apps.who.int/adolescent/second-decade/files/WHO_FWC_ MCA_14.05_spa.p

19. Ruiz $\bar{M}$. Implicaciones del uso de las redes sociales en el aumento de la violencia de género en adolescentes. Comunicación y Medios. 2015; (30): 124-141.

20. Osorio A, Aguado L. Cali, ¿cómo vamos en niñez? Priorizando la niñez en la agenda urbana [Internet]. Cali (Colombia): Pontificia Universidad Javeriana Cali; 2016 [Consultado 9 my. 2019]. Disponible en: https://www.javerianacali.edu.co/sites/ujc/files/node/field-documents/field_document_file/ informe_cali_como_vamos_ninez_2016f.pdf

21. Ramos V. Derechos sexuales y reproductivos en adolescentes de Montevideo (Tesis de maestría) [Internet]. Argentina: Facultad Latinoamericana de Ciencias Sociales FLACSO; 2011 [Consultado 9 my. 2019]. Disponible en: http://repositorio.flacsoandes.edu.ec/bitstream/10469/4969/5/ TFLACSO-2011VRB.pdf

22. Ramírez GR, Bravo PE, Vivaldi MI, Manríquez IP, Pérez TG. Acceso a anticoncepción en adolescentes: percepciones de trabajadores de la salud en Huechuraba, Chile. Rev Pana Salud Públ. $2017 ; 41$.

23. González E, Temístocles M, Montero A, Martínez V, Leyton C. Comportamientos sexuales y diferencias de género en adolescentes usuarios de un sistema público de salud universitario. Rev méd Chile [Internet]. 2007 [Consultado 3 my. 2019]; 135(10): 1261-1269. Disponible en: https:// scielo.conicyt.cl/scielo.php?script=sci_arttext\&pid=S0034-98872007001000005

24. Herrera y Cairo LJ. Apropiación de derechos sexuales y reproductivos en la adolescencia: Dimensiones de la ciudadanía. Rev Estud Géne, la Ventana. 2009; 4(30): 148-180.

25. Campero L, Kendall T, Caballero M, Mena A, Herrera C. El ejercicio de los derechos sexuales y reproductivos: un estudio cualitativo de personas heterosexuales con VIH en México. Salud Públ Méx. 2010; 52(1): 61-69.

26. Pacheco-Sánchez C, Rincón-Suárez L, Elías GE, Latorre-Santos C, Enríquez-Guerrero C, NietoOlivar J. Significaciones de la sexualidad y salud reproductiva en adolescentes de Bogotá. Salud Públ Méx [Internet]. 2007 [Consultado 3 my 2019]; 49(1): 45-51. Disponible en: http://www.scielo. org.mx/scielo.php?script=sci_arttext\&pid=S0036-36342007000100007

27. González-González FG. Conocimientos, actitudes y prácticas en salud sexual y reproductiva en jóvenes entre 14 y 25 años de edad de estratos 1 y 2 del SISBEN de un municipio del Departamento de Cundinamarca. Acta Colom Psico. 2004; (12): 59-68.

28. Grajales C, Cardona D. Actitudes de los adolescentes escolarizados frente a la salud sexual y reproductiva. Medellín (Colombia). Investigación y Educación Enferm [Internet]. 2012 [Consultado 3 my. 2019]; 30(1): 77-85. Disponible en: http://www.redalyc.org/articulo.oa?id=105224287007

29. Vinaccia S, Quiceno J, Gaviria A, Soto A, Gil M, Ballester A. Risk Sexual Behaviors for HIV Infection in Colombian Adolescents. Terapia psicológica [Internet]. 2007 [Consultado 3 my. 2019]; 25(1): 39-50. Disponible en: https://scielo.conicyt.cl/scielo.php?script=sci_abstract\&pid=S071848082007000100003\&lng=en\&nrm=iso\&tlng=en

30. Valencia C, Canaval G, Molina A, Caicedo H, Serrano L, Valencia R, Piedrahita V, Vásquez Y, Echeverri J. Servicios amigables para jóvenes: construcción conjunta ente jóvenes y funcionarios. Colombia médica [Internet]. 2010 [Consultado 3 my. 2019]; 41(1): 26-34. Disponible en: http:// colombiamedica.univalle.edu.co/index.php/comedica/article/view/682/765

31. Ministerio de la Protección Social. Decreto 2968 [Internet]. Colombia: Avance Jurídico Casa 
Editoria; 2010 [Consultado 3 my. 2019]. Disponible en: http://www.icbf.gov.co/cargues/avance/ docs/decreto_2968_2010.htm

32. Mertens D. Research and evaluation in Education and Psychology: Integrating diversity with quantitative, qualitative, and mixed methods. Thousand Oaks: Sage; 2005

33. Hernández-Sampieri R., Fernández-Collado C, Baptista LP. Metodología de la investigación. México: Editorial Mc Graw Hill; 2010

34. Willig C. Introducing qualitative research in psychology. UK: McGraw-Hill Education; 2013

35. Mieles M, Tonon G, Alvarado S. Investigacion cualitativa: el análisis temático para el tratamiento de la información desde el enfoque de la fenomenología. Universitas Humanística [Internet]. 2012 [Consultado 3 my. 2019]; (74): 195-225. Disponible en: http://www.redalyc.org/ $\mathrm{html} / 791 / 79125420009 /$

36. Ministerio de Salud. Resolución 8430 de 1993, normas científicas, técnicas y administrativas para la investigación en salud [Internet]. 4 oct. 1993 [Consultado 3 my. 2019]. Disponible en: https://www. minsalud.gov.co/sites/rid/Lists/BibliotecaDigital/RIDE/DE/DIJ/RESOLUCION-8430-DE-1993. PDF

37. Congreso de la Republica. Ley número 1090, ejercicio de la profesión de Psicología [Internet]. 6 de septiembre de 2006 [Consultado 3 my. 2019]. Disponible en: http://www.colpsic.org.co/quienessomos/ley-1090-de-2006/182

38. Jones E. Diálogos entre padres y adolescentes sobre sexualidad: discursos morales y médicos en la reproducción de las desigualdades de género. Interface-Comunicação, Saúde, Educação [Internet]. 2010 [Consultado 3 my. 2019]; 14(32): 171-182. Disponible en: https://www.scielosp.org/article/ $\mathrm{ssm} /$ content/raw/?resource_ssm_path=/media/assets/icse/v14n32/14.pdf

39. Orcasita T, Cuenca J, Montenegro J, Garrido D, Haderlein A. Diálogos y Saberes sobre Sexualidad de Padres con Hijos e Hijas Adolescentes Escolarizados. Rev Colomb Psicol [Internet]. 2018 [Consultado 3 my. 2019]; 27(1): 41-53. Disponible en: https://revistas.unal.edu.co/index.php/ psicologia/article/view/62148

40. Rodríguez A, Sanabria G, Contreras ME, Perdomo B. Estrategia educativa sobre promoción en salud sexual y reproductiva para adolescentes y jóvenes universitarios. Revista Cubana de Salud Pública. 2013 [Consultado 22 oct. 2017]; 39(1): 161-174. Disponible en: http://scielo.sld.cu/scielo. php?pid=S0864-34662013000100015\&script=sci_arttext\&tlng=en

41. Orejerena Y, Blanco MA. Conocimientos, actitudes y prácticas en sexualidad en jóvenes universitarios de Colombia, revisión sistemática de la literatura (tesis de pregrado). 2015 [Consultado 22 oct. 2017]. Disponible en: http://udes-dspace.metabiblioteca.com/handle/001/40

42. Juárez-Herrera y Cairo, L. Apropiación de derechos sexuales y reproductivos en la adolescencia: dimensiones de la ciudadanía. La ventana. Revista de estudios de género. 2009 [Consultado 23 nov. 2017]; 4(30): 148-180. Disponible en: http://www.scielo.org.mx/scielo.php?script=sci_ arttext\&pid=S1405-94362009000200007\&lng=es\&tlng=es 